\title{
RESEARCH
}

Open Access

\section{Combined topical and systemic administration with human adipose-derived mesenchymal stem cells (hADSC) and hADSC-derived exosomes markedly promoted cutaneous wound healing and regeneration}

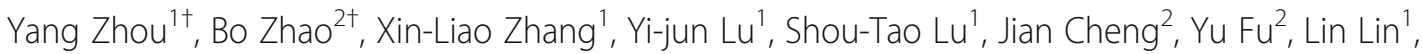
Ning-Yan Zhang ${ }^{2}$, Pei-Xin Li', Jing Zhang ${ }^{2,3,4^{*}}$ and Jun Zhang ${ }^{1,3,4^{*}}$

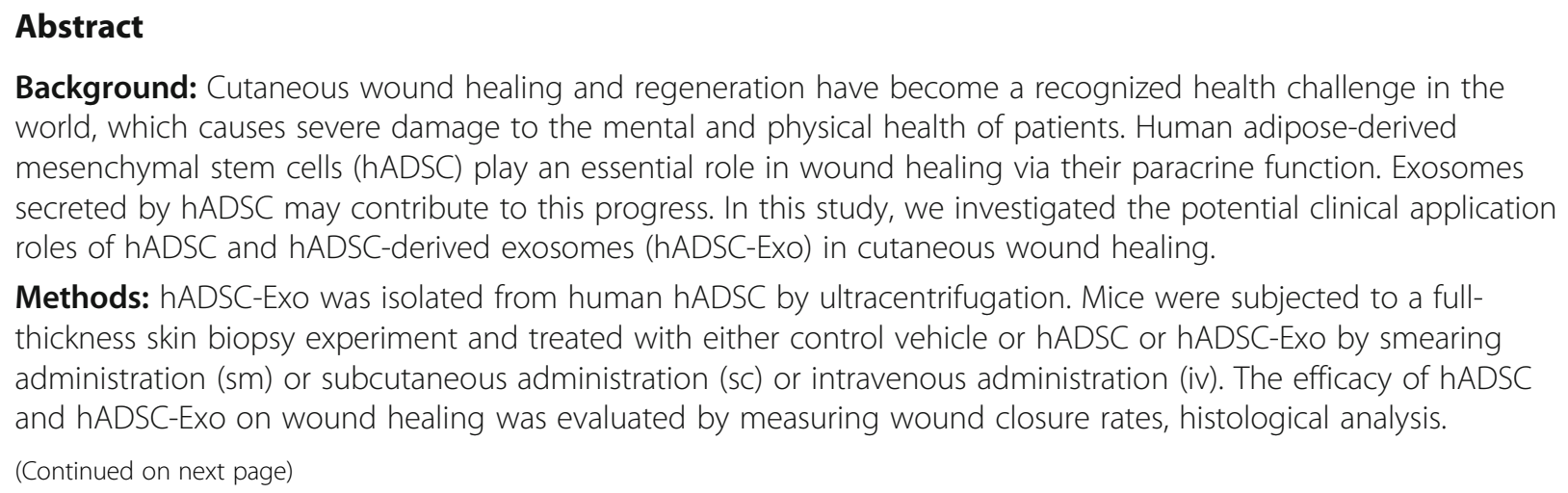
world, which causes severe damage to the mental and physical health of patients. Human adipose-derived mesenchymal stem cells (hADSC) play an essential role in wound healing via their paracrine function. Exosomes secreted by hADSC may contribute to this progress. In this study, we investigated the potential clinical application roles of hADSC and hADSC-derived exosomes (hADSC-EXo) in cutaneous wound healing.

Methods: hADSC-Exo was isolated from human hADSC by ultracentrifugation. Mice were subjected to a fullthickness skin biopsy experiment and treated with either control vehicle or hADSC or hADSC-Exo by smearing administration (sm) or subcutaneous administration (sc) or intravenous administration (iv). The efficacy of hADSC and hADSC-Exo on wound healing was evaluated by measuring wound closure rates, histological analysis.

\footnotetext{
* Correspondence: zhangjing@tongji.edu.cn; junzhang@tongji.edu.cn

${ }^{\dagger}$ Yang Zhou and Bo Zhao contributed equally to this work.

${ }^{2}$ Key Laboratory of Spine and Spinal Cord Injury Repair and Regeneration of Ministry of Education, Stem Cell Translational Research Center of Tongji Hospital, School of Life Science and Technology, Tongji University, 389 Xincun Road, Shanghai 200065, China

${ }^{1}$ Research Center for Translational Medicine, Shanghai East Hospital, School of Medicine, Tongji University, Shanghai 200092, China

Full list of author information is available at the end of the article
}

(c) The Author(s). 2021 Open Access This article is licensed under a Creative Commons Attribution 4.0 International License, which permits use, sharing, adaptation, distribution and reproduction in any medium or format, as long as you give appropriate credit to the original author(s) and the source, provide a link to the Creative Commons licence, and indicate if changes were made. The images or other third party material in this article are included in the article's Creative Commons licence, unless indicated otherwise in a credit line to the material. If material is not included in the article's Creative Commons licence and your intended use is not permitted by statutory regulation or exceeds the permitted use, you will need to obtain permission directly from the copyright holder. To view a copy of this licence, visit http://creativecommons.org/licenses/by/4.0/. The Creative Commons Public Domain Dedication waiver (http://creativecommons.org/publicdomain/zero/1.0/) applies to the data made available in this article, unless otherwise stated in a credit line to the data. 
(Continued from previous page)

Results: Combined application of local hADSC-Exo smearing and hADSC/hADSC-Exo intravenous administration offered the additional benefit of promoting wound healing, accelerating re-epithelialization, reducing scar widths, and enhancing angiogenesis and collagen synthesis. Either topical application of hADSC-Exo or systemic administration with hADSC/hADSC-Exo appeared more effective in stimulating cell proliferation, inhibiting cell apoptosis and inflammation, and promoting skin elasticity and barrier integrity, with increased genes expression of PCNA, VEGF, collagen III, Filaggrin, Loricrin, and AQP3, with decreased genes expression of TNF-alpha.

Conclusion: Our findings suggest that the combined administration of hADSC/hADSC-Exo can facilitate cutaneous wound healing and reduce scar formation. These data provide the first evidence for the feasibility of smearing of hADSC-Exo as a cell-free therapy in treating cutaneous wounds, and the potential clinical value of combined administration of hADSC/hADSC-Exo.

Keywords: Adipose mesenchymal stem cells, Exosome, Wound healing

\section{Background}

Cutaneous wound healing is an intricate and highly orchestrated physiological process, including hemostasis, inflammation, tissue formation, and tissue remodeling [1-3]. The normal wound healing process is disrupted and prolonged in some disease conditions resulting in chronic non-healing wounds, for instance, diabetic ulcers or pathological scars, e.g., keloid scars [2, 4, 5]. This has caused severe damage to the patients' economic situation, as well as their mental and physical health. Thus, it is urgent for us to find an effective way to shorten the wound's healing time and reduce scar formation. Previous studies have shown that hADSC can enhance wound healing, inhibit scar formation, and resist photoaging through a paracrine mechanism [6-11]. Recently, multiple studies have documented that exosomes play a crucial role in paracrine mechanisms. Exosomes have received much attention in basic research and clinical utility [12, 13].

Exosomes, $30-200 \mathrm{~nm}$ vesicles originate from the rough endoplasmic reticulum, encapsulate various active substances [14, 15]. Exosomes are significant carriers of signals between cells. They play vital roles in intercellular communication via transferring specific DNAs, proteins, microRNAs, lipids, mRNAs, and other signaling molecules to target cells [15-17]. Exosomes are a vital mediator of paracrine function, found in many body fluids, including the saliva, serum, and urine. A growing body of evidence has demonstrated that MSC-derived exosomes' therapeutic potential for tissue responses to injury, infection, and disease [15, 18-21]. Research documents that exosomes show similar capacities to host cells and exhibit no apparent side effects, e.g., malignant transformation, vascular obstructive risks, and immunogenicity, demonstrating that therapy based on exosomes is considerably safe and promising for tissue regeneration therapy compared to the direct utilization of cells [22-26].

Herein, we used a mouse model to explore the effects of hADSC and hADSC-Exo on repairing wound skin.
We found that the topical medication of smearing hADSC-Exo is more beneficial for promoting skin damage repair than the subcutaneous injection of hADSC or hADSC-Exo. This is mainly because hADSCExo can promote cell proliferation, angiogenesis, collagen synthesis, and skin barrier function repair, which benefits wound healing. For systemic delivery, local administration combined therapy with the intravenous injection of hADSC and hADSC-Exo offered additional benefit over either therapy alone to enhance cutaneous regeneration. Herein, we, for the first time, access the therapeutic effect of hADSC and hADSC-Exo for wound healing and provide simple and effective clinical treatment suggestions for acute and chronic wounds.

\section{Methods and materials \\ Isolation and identification of human adipose mesenchymal stem cells (hADSC)}

Human adipose tissue samples were obtained from a healthy female donor with the donor's written informed consent as approved by the East Hospital Affiliated to Tongji University. This healthy female donor is 30-35 years of age with HIV-negative, HBV-negative, HCVnegative, $\mathrm{CMV}$-negative, EBV-negative, and TP-negative. hADSC were isolated from adipose tissue as previously reported, and they were positive for CD73, CD90, and CD105, and negative for CD45 and HLA-DR [22]. In brief, adipose tissues were washed 2-3 times with sterile normal saline and then centrifuged at $1500 \mathrm{rpm}$ for 10 min. After centrifugation, the liquid was divided into three layers. The upper layer of floating adipocytes was treated with collagenase type I for $30 \mathrm{~min}$ at $37^{\circ} \mathrm{C}$ with intermittent shaking. The cell pellets were separated by centrifugation at $1500 \mathrm{rpm}$ for $10 \mathrm{~min}$ and then suspended in complete medium containing Minimum Essential Medium $\alpha$ (MEM) (Gibco,USA) and 10\% UltraGRO ${ }^{\mathrm{Tm}}$-Advanced (Helios Bioscience, USA) supplemented with 100 $\mathrm{U} / \mathrm{mL}$ penicillin-streptomycin (Gibco, USA). The primary hADSC were cultured for 4-5 days until they reached 
confluence (defined as passage 0 ). The cells were then harvested by $0.25 \%$ trypsin-EDTA (Invitrogen, USA) and diluted 1:2 to $1: 3$ and plated for subculture, and the cell used in experiments during the third-seventh passages. After 3-7 passages, the hADSC were harvested and stained with fluorescence-conjugated antibodies (BD Biosciences): anti-CD73-PE, anti-CD105-BV421, anti-CD90-APC, antiCD45-FITC, and anti-HLA-DR-PE-Cy5-conjugated. Isotype control IgG was used to stain the cells as a control. After two washes with PBS, the cells were analyzed by using a flow cytometer (BD FACSVerse ${ }^{\mathrm{Tw}}$, Becton-Dickinson, San Jose, CA, USA), and the data analysis was conducted using the Flowjo software (Tree Star Inc., USA).

For the adipogenic, osteogenic, and chondrogenic differentiation, hADSC were incubated into adipogenic, osteogenic, and chondrogenic differentiation medium (Cyagen, China) for 3 weeks. For adipogenesis, cell pellets were stained with oil red $\mathrm{O}$ to detect the intracellular accumulation of lipid vacuoles. Osteogenesis was demonstrated by the formation of bone matrix assessed by Alizarin Red S staining. Chondrogenesis was confirmed by the secretion of sulfated glycosaminoglycans stained with Alcian Blue.

\section{Isolation and identification of hADSC-Exo}

Exosomes were isolated from culture supernatant of hADSC as demonstrated previously [25]. Briefly, the culture media of confluent hADSC was collected and centrifuged at $300 \times g$ for $10 \mathrm{~min}$ to remove cells. Transfer the supernatant from the centrifugal tube to the new centrifugal tube and centrifuge at $2000 \times g$ for $10 \mathrm{~min}$. To remove cell debris, transfer the supernatant to the new centrifugal tube again and centrifuge at $10,000 \times$ g for 30 $\mathrm{min}$. After filtered through a $0.2-\mu \mathrm{m}$ filter, the supernatant was again collected and centrifuged at $100,000 \times g$ for $2 \mathrm{~h}$ at $4{ }^{\circ} \mathrm{C}$. The supernatant was then discarded because the pellet now contained exosomes. Exosomes were resuspended in $1 \times \mathrm{PBS}$ and stored at $-80^{\circ} \mathrm{C}$ for the following experiments. Exosomes were quantified with the total exosome protein assay. The quantification of the total exosome protein was addressed in the methods of Western Blot. Generally, 100 $\mathrm{g}$ g exosomes could be isolated from $20 \mathrm{ml}$ culture medium of 3-7 passages $10^{\times 9}$ hADSC. The morphology of exosomes was observed under a transmission electron microscope (TEM, FEI, USA). The exosomes were diluted with $1 \times$ PBS (10-fold), filtered the mixture into a new centrifuge tube with a $0.22 \mu \mathrm{m}$ filter, and then subjected to Nanosight tracking analysis (NTA, Malvern, USA).

\section{In vivo wound healing experiments in an animal model}

All procedures were approved by the Animal Research Committee of Tongji University. Male ICR mice (7 weeks old, weighing 28-35 g) maintained under specific pathogen-free (SPF) conditions, which were purchased from Shanghai SLAC Laboratory Animal Co., Ltd., were used in this study. After shaving the mice, we created a $1.5 \times 1.5 \mathrm{~cm}$ full-thickness wound on the back of mice to confirm the effects of hADSC and hADSC-Exos on cutaneous wound healing. Mice were randomly divided into 5 different groups: untreated group (control group), rhEGF local smearing group (positive group), $1 \times 10^{6}$ hADSC in $200 \mu \mathrm{l}$ PBS subcutaneous administration (sc) group (hADSC-sc group), $200 \mu$ g exosome in $200 \mu \mathrm{lBS}$ subcutaneous administration group (hADSC-Exos-sc group), and $200 \mu \mathrm{g}$ exosome in $200 \mu \mathrm{l}$ PBS local smearing group (hADSC-Exos-sm group). The mice were housed individually and were treated with above these measures once a day. The wound areas were measured at days $0,3,7,10$, and 12 and calculated with image analysis software. Mice were killed at day 12 after surgery. Half of the wound skin tissues were collected for immunohistochemistry staining and western blot analysis.

To further confirm the relationship between different frequencies and the optimal protocol for hADSC-Exo$\mathrm{sm}$, we divided the mice into three groups and hADSCExo-sm was carried out by three different frequency of administration for up to 12 days of continuous administration as follows: Qd: once a day, Bid: twice a day, and Tid: three times a day. A single application dose of hADSC-Exo-sm is $200 \mu \mathrm{g}$ in $200 \mu \mathrm{l}$ PBS. The wound areas were measured at days $0,3,7,10$, and 12 and calculated with image analysis software. Wound skin tissues were collected on day 12 following treatment for immunohistochemistry staining. To further compare the effects of topical hADSC-Exo-sm treatment and systemic medication, hADSC, hADSC-Exo, and PBS were injected by tail vein injection 1 day after wound modeling. Similarly, the wound tissues were collected and stained for immunohistochemical analysis.

\section{Histological analysis}

The skin at the wound site of each group of mice was cut and fixed. For the histological evaluation, the worst part of each wound healing condition was selected for analysis. Tissue sections were fixed overnight in 10\% buffered formalin at room temperature. Next, the samples were transferred to $70 \%$ ethanol for an additional $48 \mathrm{~h}$ and then embedded in paraffin. Serial sections from the middle of the wound were made in a $5-\mu \mathrm{m}$ crosssection.

The sections were stained with hematoxylin and eosin H\&E and Masson's trichrome. Immunohistochemistry was performed as described above. The dewaxed sections were washed in PBS, and the endogenous peroxidase activity was quenched by immersion in $2 \%(\mathrm{v} / \mathrm{v})$ hydrogen peroxide for $5 \mathrm{~min}$. The antigen retrieval was repaired by incubation with sodium citrate buffer for 30 
min. After washing in PBS, the sections were sealed with $1.5 \%$ goat serum at room temperature for $30 \mathrm{~min}$ and then incubated with primary antibodies provided in Table 1 , overnight at $4{ }^{\circ} \mathrm{C}$, and then washed with PBS and incubated with respective secondary antibody (goat anti-rabbit or goat anti-mouse, ZSGB-BIO, China) for $20 \mathrm{~min}$ at room temperature. Immunohistochemical staining was developed using the DAB substrate system (DAKO, Denmark). Images were collected using a BX53 microscope (Olympus, Japan).

IHC image analysis was performed using Image-Pro Plus 6.0 software. Three animals per group were analyzed for immunohistochemical staining. For each sample, at least 5 fields ( $\times 400$ magnification) were randomly selected for analysis. For CD14, VEGF, TNF- $\alpha$, PCNA, Ki67, IL-6, CD68, CD19, and Cleaved Caspase3 analyses, data are represented as the percentage of positive cell numbers divided by total cell numbers. For collagenous fiber in Masson, collagen I, collagen III, AQP3, and Loricrin burden analyses, data are reported as the mean density (IOD/Area) of positively stained regions. The average number of CD31-positive small vessels were manually counted in five random fields. All histological assessments were performed by two independent observers.

\section{Western blotting}

Exosomes were lysed with RIPA lysis buffer (ThermoFisher, USA) supplemented with the protease inhibitor cocktail and phosphatase inhibitor mini-tablets (Pierce, USA). The lysate was centrifuged at $3000 \mathrm{rpm}$ for 15 min at $4{ }^{\circ} \mathrm{C}$, and the supernatant was quantitated using the Micro Bicinchoninic Acid (BCA) Protein Assay Kit (Pierce). The total $40 \mu$ g protein was resolved by SDSPAGE on $10 \%$ polyacrylamide gels and was transferred

Table 1 The dilutions of the primary antibodies used for $\mathrm{IHC}$

\begin{tabular}{llll}
\hline Protein name & Dilution & Company & Area \\
\hline Collagen I & $1: 1000$ & Abcam & Cambridge, UK \\
Collagen III & $1: 2000$ & Abcam & Cambridge, UK \\
Ki67 & $1: 400$ & CST & MA, USA \\
PCNA & $1: 1000$ & Boster & CA, USA \\
Cleaved-Caspase 3 & $1: 100$ & Abcam & Cambridge, UK \\
VEGF & $1: 500$ & Abcam & Cambridge, UK \\
CD31 & $1: 1000$ & Abcam & Cambridge, UK \\
Filaggrin & $1: 500$ & Abcam & Cambridge, UK \\
Loricrin & $1: 200$ & Abcam & Cambridge, UK \\
AQP3 & $1: 500$ & Abcam & Cambridge, UK \\
TNF-a & $1: 200$ & Boster & CA, USA \\
CD14 & $1: 100$ & Abcam & Cambridge, UK \\
CD19 & $1: 200$ & ZSGB-BIO & Beijing, China \\
CD68 & $1: 200$ & Boster & CA, USA \\
\hline
\end{tabular}

onto Hybond-P polyvinylidene difluoride (PVDF) membrane (Millipore). The membranes were incubated with antibodies overnight at $4{ }^{\circ} \mathrm{C}$ and further incubated with the appropriate horseradish peroxidase (HRP)-conjugated secondary antibodies (Abcam, Cambridge, UK) for $1 \mathrm{~h}$ at room temperature. The blots were visualized by enhanced chemiluminescence (ECL) detection reagents (Bio-Rad, USA). Images were documented, and band density was analyzed by Amersham Imager 600 (GE). The antibodies were used in this study as follows: antiCD9 (Abcam, Cambridge, UK) and anti-CD63 (Abcam, Cambridge, UK).

\section{Statistical analysis}

Statistical analysis. Statistical analysis was performed using Graphpad Prism software. Two-tailed Student's $t$ test was applied when there were only two groups of samples. In the case of more than two groups of samples, one-way ANOVA was used with one condition, and two-way ANOVA was used with more than two conditions. ANOVA analysis was followed by post hoc Bonferroni's correction for multiple comparisons. $p<0.05$ was taken as statistically significant; $" p$ value $<0.05$, " $p$ value $<0.01, * * * p$ value $<0.001$, and $* * * * p$ value $<0.0001$. The data displayed a normal distribution. The estimated variance was similar between experimental groups. Data are presented as the mean \pm SD or \pm SEM, as indicated in the figure legends.

\section{Results and discussion}

\section{Characterization of hADSC and hADSC-Exo}

hADSC cells were grown as described in the "Methods" section and had typical fibroblastic morphology as indicated in Fig. 1a. The cells exhibited adipogenic, osteogenic, and chrondrogenic differentiation properties as illustrated by the oil red $\mathrm{O}$ staining of the adipocytes, Alizarin Red S staining of the osteoblasts, and Alcian Blue staining of chrondrogenic, and respectively, as indicated in Fig. 1b-d. The flow cytometry data demonstrated that hADSC cells were remarkably positive for MSC surface biomarkers consisting of CD105, CD73, and CD 90, and negative for HLA-DR and CD45.

Exosomes were extracted from the supernatants of hADSC by ultra-spinning. The electron microscopy data demonstrated that hADSC-Exo had a saucer-shaped morphology, with diameters ranging between 30 to 100 $\mathrm{nm}$ (Fig. 1f), consistent with previously reported exosomes. Nanosight analysis was employed to explore the particle concentration, size distribution, and dynamic tracking, as indicated in Fig. 1g. The western blotting data revealed some exosome biomarkers, including CD9 and CD63, expressed in exosomes, further validating their identity as exosomes. Altogether, these data demonstrated 


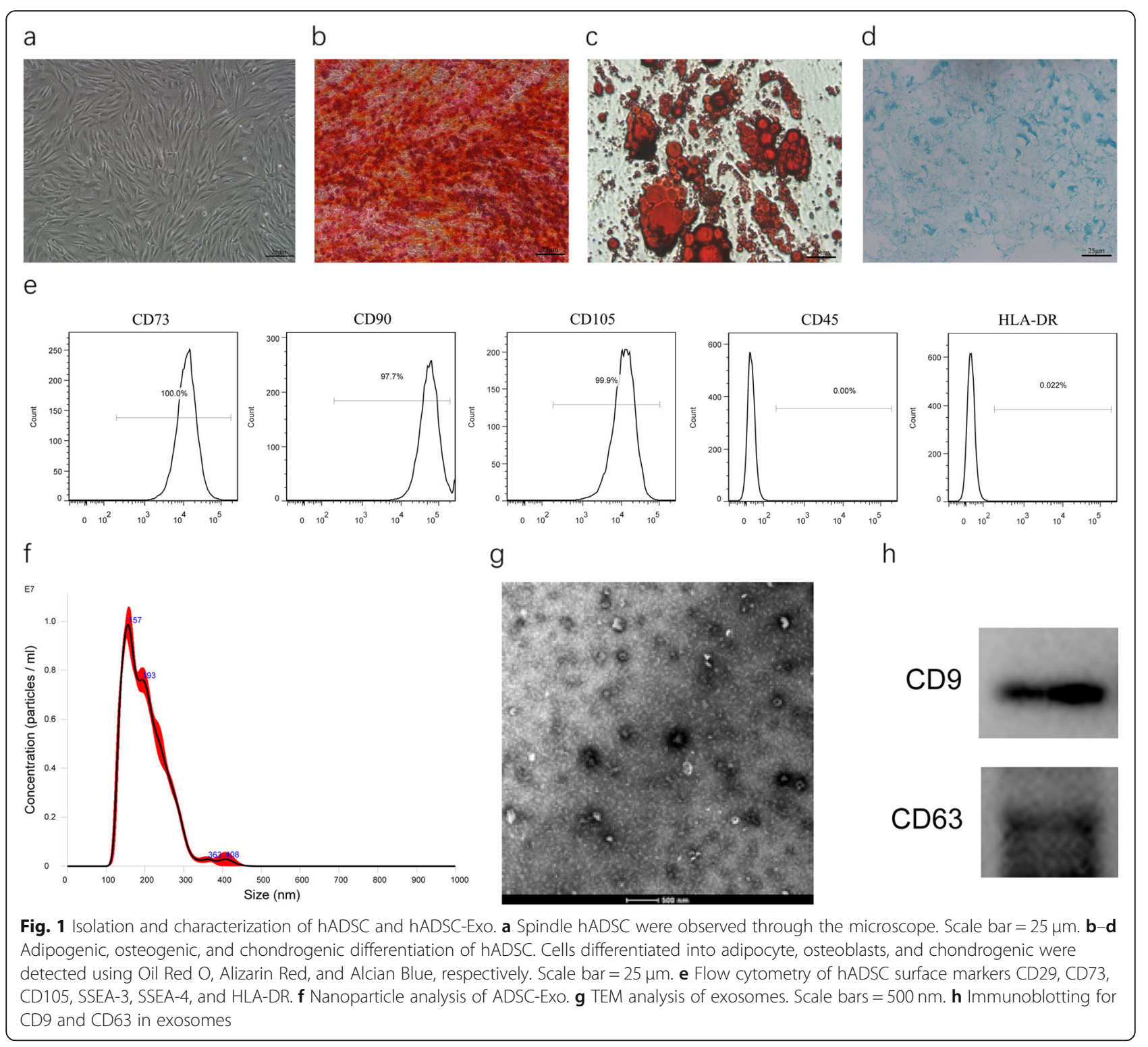

that hADSC-Exo was isolated successfully and was consistent with defined exosomes.

\section{hADSC-Exo-sm promotes cutaneous wound healing}

To evaluate the effect of hADSC and hADSC-Exo on cutaneous wound healing, we apply the mice model. The full-thickness cutaneous wounds were created on the dorsal skin areas of male ICR mice, followed by the treatment of hADSC-sc, hADSC-Exo-sc, and hADSCExo-sm on the wound sites once a day for 14days (Fig. 2a). As a positive control, Recombinant Human Epidermal Growth Factor Hydro Gel $(1 \mathrm{~g} / \mathrm{cm}$, rhEGF, trade name $\mathrm{YiFu}$ ) was locally smearing administered once daily. As in Fig. 2b, c, both subcutaneous administration and smearing administration of hADSC and hADSC-Exo significantly promote the cutaneous wounds closure rate compared to the controls. Notably, the hADSC-Exo-sm group and the rhEGF group appeared to have a significantly better velocity of the wound healing process than the other groups on days 7,10 , and 12 post wounding. Surprisingly, the better epithelial tissue was observed in the hADSC-Exo-sm groups in HE staining observation (Fig. 2d). Masson staining (Fig. 2e) of tissue sections also illustrated that wounds treated with hADSC-Exo-sm exhibited well-reorganized collagen fibers compared to other post-wounding wounds, implying that hADSC-Exo-sm treated wounds exhibited remarkably shorten healing time, as well as narrower scars.

We performed further in vivo investigations to establish whether hADSC-Exo-sm treatment can accelerate the wound closure rate in a dose-dependent manner. To better simulate the local clinical administration of skin 


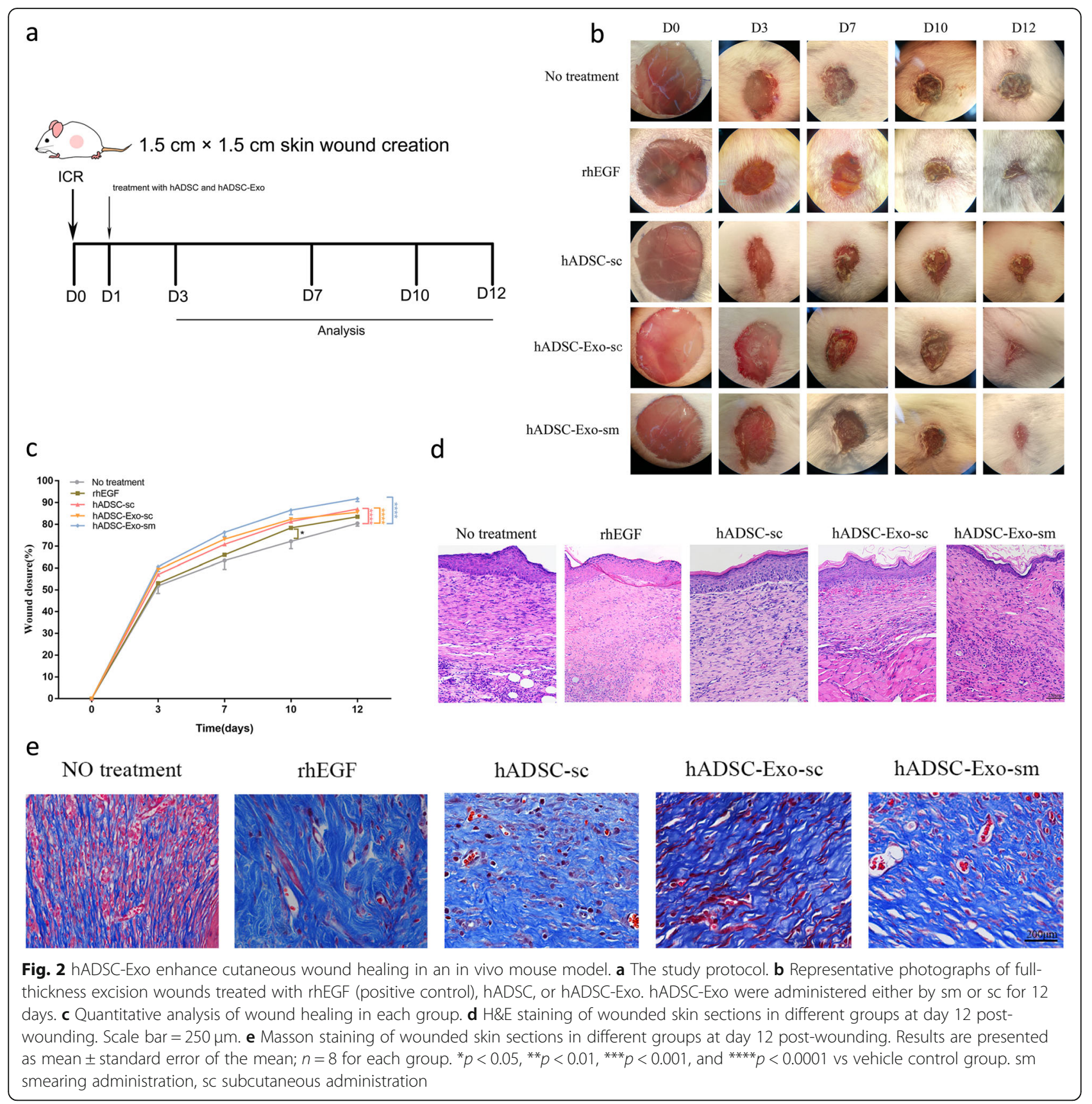

wounds, we smeared with three different administration frequencies of hADSC-Exo instead of just increasing the hADSC-Exo dose. The rhEGF was used as a positive control. hADSC-Exo-sm was carried out by three different administration frequencies for up to 12 days of continuous administration: Qd: once a day, Bid: twice a day, and Tid: three times a day. As indicated in Fig. 3a, b, the full-thickness wound mice receiving treatment of hADSC-Exo-sm three times a day exhibited a higher wound closure rate than that treated with other administration at days $3,7,10$, and 12 post-wounding. Besides, the wounds treated with hADSC-Exo-sm three times a day showed markedly enhanced re-epithelialization at day 12 post-wounding (Fig. 3c). Meanwhile, the greater extent of angiogenesis, the more enhanced proliferation, and the better-suppressed inflammation were reported in the wound sites treated with a three-times-a-day frequency of hADSC-Exo-sm (Fig. 3d-i).

\section{hADSCs-Exo-sm promoted collagen synthesis, skin barrier repair in vivo}

Further in vivo studies were performed to determine whether hADSC-Exo can affect collagen synthesis ability in the wound site. As indicated in Fig. 4a, higher 
collagen maturity was reported via Masson staining in wounds treated with ADSC-Exo-sm than the other groups. IHC staining (Fig. 4b, c) of collagen I and collagen III were conducted to visualize the effect of hADSCExo-sm on fibroblasts. Similar findings were reported that hADSC-Exo-sm promoted the deposition of collagens. With the increase of hADSC-Exo smearing administration frequency, the wound area showed a greater extent of collagen maturity, and the ratios of collagen I to collagen III were decreased. Scarless healing fetal wounds aggregate more collagen type III relative to scarring adult wounds with a higher proportion of type I collagen deposition [27-30]. These findings precisely demonstrate that hADSC-Exos modulate the deposition and organization of collagen to enhance a scarless pattern.

The epithelium's outer layer is referred to as the stratum corneum, which accounts for the permeability of the skin barrier and the resilience of the cornified epithelium. Filaggrin contributes to forming relevant components for $\mathrm{pH}$ maintenance, moisture, and skin protection against microbial agents. Loricrin is an insoluble protein that forms inter-and intra-protein crosslinks that are highly resistant to proteolysis and stabilize and strengthen the epithelium structure. AQP3 is the predominant aquaporin in the human skin, located in the epidermis's basal layer [31] and the stratum corneum [32]. These skin barrier proteins are essential to control the epidermis' selective permeability to build a barrier against the external environment. IHC analysis results showed that hADSC-Exo-sm three times a day improves skin hydration and epidermal permeability barrier function by stimulating the expression of Filaggrin, Loricrin, and AQP3 (Fig. 4d, e).

\section{Systemic administration facilitate the cutaneous wound healing}

To investigate the effect of systemic administration for cutaneous wound healing, we performed the intravenous administration with hADSC and hADSC-Exo at $24 \mathrm{~h}$ posting-wounding. We found that iv administration of hADSC and hADSC-Exo significantly accelerated wound closure and reduced scar formation (Fig. 5a, b). The wounds treated with iv of hADSC/hADSC-Exo and topical application of hADSC-Exo-sm, compared to other groups, exhibited remarkably enhanced re-epithelialization as indicated in Fig. 5c. Masson staining (Fig. 5d) and IHC staining of Col-I and Col-III (Fig. 5e, f) demonstrated that hADSC/hADSC-Exo iv and hADSC-Exo-sm treated wounds exhibited well-reorganized collagen fibers relative to the other treatment groups post-wounding. The findings illustrated that exosome treatment increased collagen III content in the wound's bed, and it is organized in a subtle reticular trend. hADSC-Exo sm or hADSC-Exo iv changed the ratio of collagen I to collagen III from a scar-enhancing higher ratio to an anti-scarring lower ratio and decreased the degree of scarring.

In contrast with the other groups, the expression contents of CD31 and VEGF were significantly increased, demonstrating a valid promotion of angiogenesis at the site of wound with hADSC/hADSC-Exo iv and hADSCExo-sm treatment (Fig. 5g, h). The numbers of Ki67 and PCNA cells increased in the skin lesions while the number of C-Caspase 3 cells decreased (Fig. 5i, j), indicating that the hADSC/hADSC-Exo iv and hADSC-Exo-sm treatment can promote cell proliferation and inhibit apoptosis. The hADSC/hADSC-Exo iv and hADSC-Exosm treatment also remarkably reduced expression of the inflammatory cytokines consisting of CD14, CD19, CD68, and TNF- $\alpha$ in skin lesions of ICR mice (Fig. $5 \mathrm{k}$, 1). All the above results revealed that hADSC/hADSCExo iv and hADSC-Exo-sm treatment could effectively accelerate the skin wound healing and remodeling the skin structure, which provides reliable research data for future clinical application of hADSC/hADSC-Exo in the cutaneous wound.

\section{Discussion}

Herein, we investigated and compared the therapeutic effects of hADSC and hADSC-Exo on wound healing and cutaneous regeneration. Our findings illustrated for the first time that a combination treatment of hADSC and hADSC-Exo significantly promotes cutaneous wound healing, collagen synthesis, vascularization, and skin barrier repair at wound sites in a mouse fullthickness skin defect model. For topic application, hADSC-Exo smearing improves wound healing more than hADSC. For systemic delivery, combined therapy with the intravenous injection of hADSC-Exo and hADSC offered additional benefit over either therapy alone to enhance cutaneous regeneration.

Studies have opined that the primary mechanism responsible for therapy involving stem cell transplantation is paracrine, with exosomes actively contributing to stem cells' paracrine properties [33, 34]. Induced pluripotent stem cells (iPSCs), iPSC-derived mesenchymal stem cells (iMSCs), and MSC-derived exosome therapeutic effect in cutaneous wound healing have been reported previously [35-37]. Some preclinical experiments have shown that exosomes from iPSC or iMSCs exert superior functions compared with exosomes derived from MSCs (MSC-Exo) [37]. Although iPSCs possess the self-renewal ability and can differentiate into any types of adult cell, they are not linked to any ethical issues and can serve as a great arsenal of stem cell transplantation therapy; they are possibly tumorigenic [38]. Conversely, the proliferation along with the differentiation abilities of MSCs are limited after numerous passages in culture $[39,40]$. Mounting research 
a

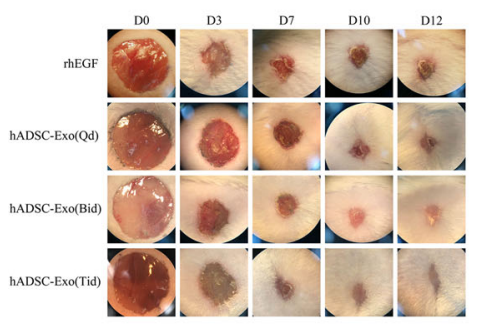

C
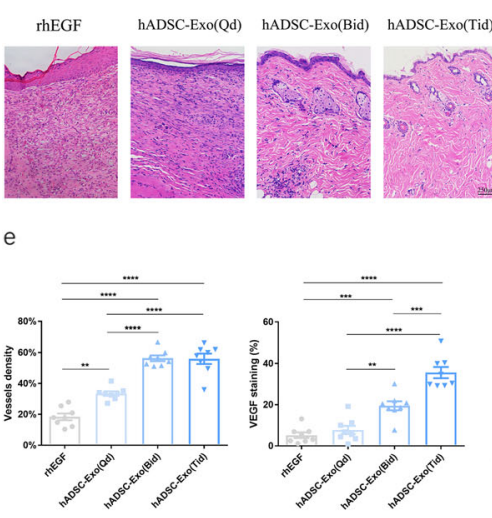

hADSC-Exo(Qd) hADSC-Exo(Bid) haDSC-Exo(Tid)

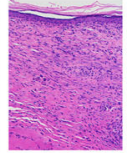

b

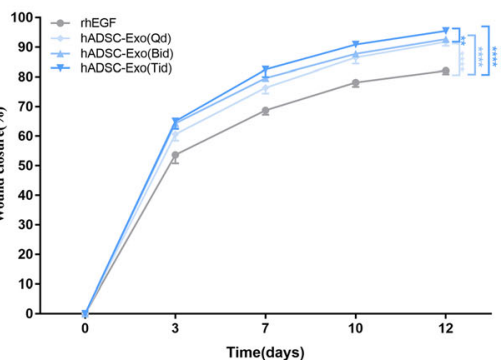

d
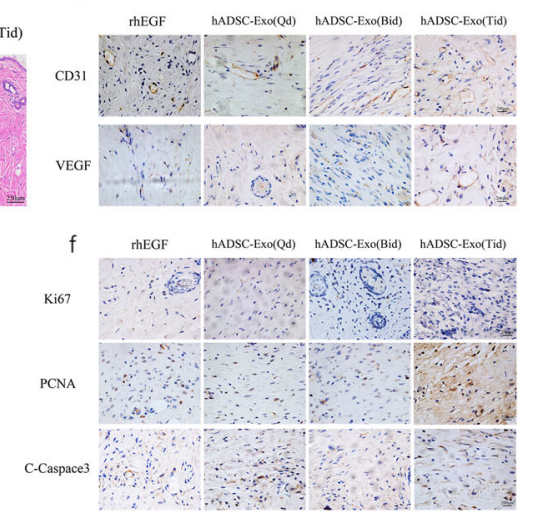

g

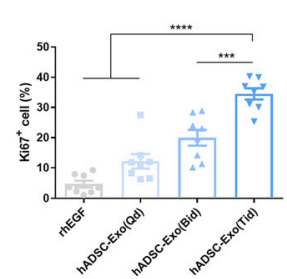

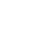






hADSC-originated exosomes mimic the function of the host cells and could effectively avert the risk of tumor development, immune-induced rejection, limited survival of cells, and loss of function or senescence-triggered genetic instability. Thus, herein, combined therapy with hADSC and hADSC-Exo was first adopted to examine their impacts in cutaneous wound healing in a mouse fullthickness skin defect model.

The typical process of skin regeneration can be summarized into four overlapping phases, namely the inflammation stage, the angiogenesis stage, the proliferation stage consisting of cell proliferation along with reepithelization, and the final remodeling stage. Several studies currently demonstrated the role of MSC-exosomes in the above three stages of wound healing and cutaneous regeneration. Inflammation constitutes the initial response of the four phrases of the typical wounding repair. We found that hADSC and hADSC-Exo can alleviate the inflammatory response resulting from numerous stimuli by downregulating cytokines and chemokines, like tumor necrosis factor (TNF)- $\alpha$, interleukin (IL)-6, CD14, CD19, and CD68. During the proliferation stage, neoangiogenesis, collagen deposition, re-epithelialization, and wound contraction concur. Neoangiogenesis formation is a pivotal step in wound healing, as well as tissue repair [42]. The results showed that the treatment of hADSC-Exo could increase 

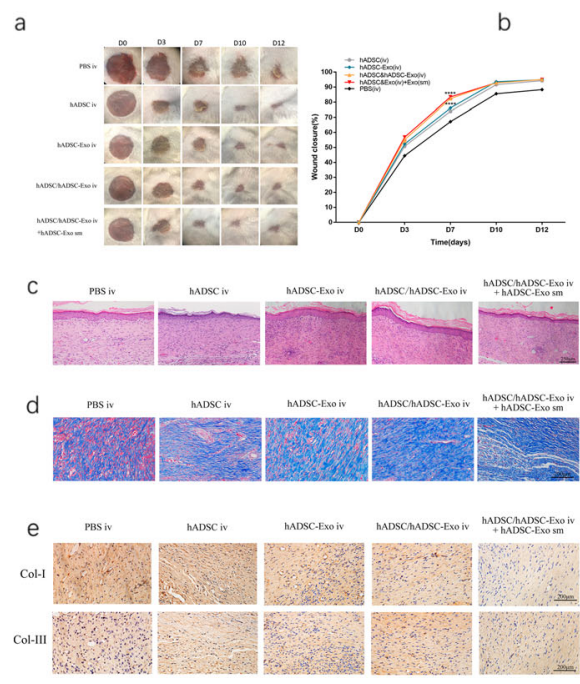

$f$
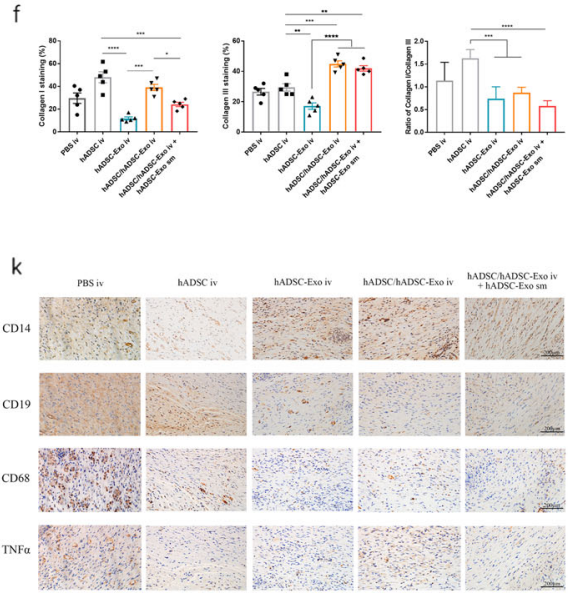

g

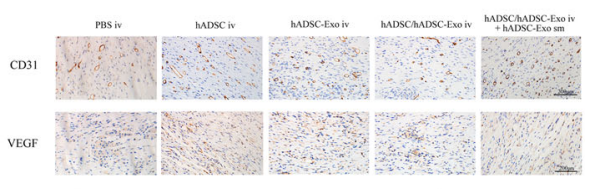

$\mathrm{h}$
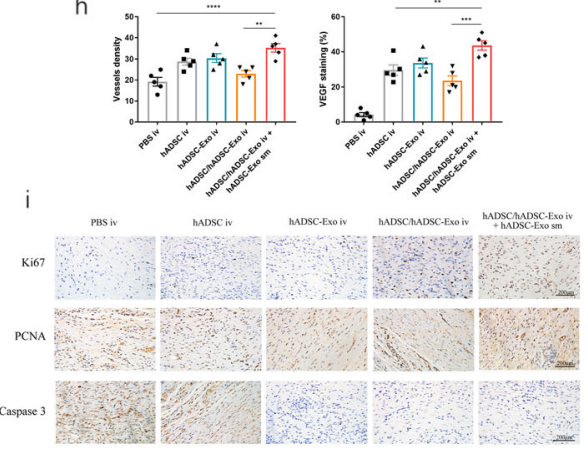

j

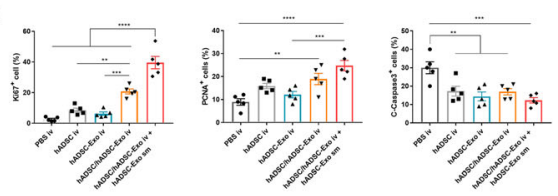

I
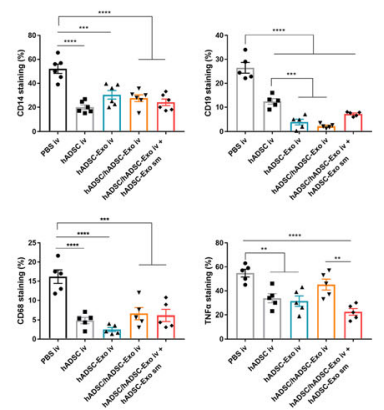

Fig. 5 Therapeutic effects of hADSC-Exo in an in vivo mouse model. a Representative photographs of full-thickness excision wounds treated with hADSC iv and hADSC-Exo iv with or without hADSC-Exo sm. b Quantitative analysis of wound healing in each group. c Histological structure of wounded skin in different groups. Scale bar $=250 \mu \mathrm{m}$. d Masson staining of wounded skin sections in different groups at day 12 post-wounding. Scale bar $=200 \mu \mathrm{m}$. e The IHC of collagen-I and collagen-III in wounded skin sections on day 12 post-wounding. Scale bar $=200 \mu \mathrm{m}$. $\mathbf{f}$ Quantitative analysis of collagen I, collagen III, and the ratio of collagen I/collagen III. g Representative images of immunohistochemical results of CD31 and VEGF. Scale bar $=200 \mu \mathrm{m}$. $\mathbf{h}$ Quantitative analysis of the number of mature blood vessels. Quantitative analysis of the positive cells in the membrane tissues. i Representative images of immunohistochemical results of Ki67, PCNA, and C-Caspase3. Scale bar $=200 \mu \mathrm{m}$. $\mathbf{j}$ Quantitative analysis of the positive cells in the skin tissues. $\mathbf{k}$ Representative photographs of CD14, CD19, CD68, and TNF-alpha immunostaining. Scale bar = $200 \mu \mathrm{m}$. I Quantification of CD14+, CD19+, CD68+, and TNF-alpha+ IHC stained tissues. Results are presented as mean \pm standard error of the mean; $n=5$ for each group. ${ }^{*} p<0.05,{ }^{* *} p<0.01,{ }^{* * *} p<0.001$, and ${ }^{* * *} p<0.0001$ vs vehicle control group

the expression of angiogenesis-associated molecules consisting of VEGF and CD31. The results also showed that hADSC-Exo promoted wound healing, enhanced reepithelialization, elevated the expression of Ki67, PCNA, and decreased the expression of C-Caspase 3. The remodeling of the extracellular matrix normally lasts for 2 weeks to more than 1 year. The remodeling stages of wound healing are closely linked to the production of the ECM and its reorganization, which is vital in establishing the scarring extent. The synthesis and degeneration of collagen constitute the key to ECM reconstruction. The narrower scars were observed in wounds treatment with hADSC-Exo(sm) three times a day compared with other groups in our study, and our findings revealed that hADSC-Exo increased the ratios of collagen III to collagen I in the late stage to suppress scar tissue formation, which is one of the prospective mechanisms of reducing scar formation. At last, we investigate the effect of hADSC-Exo on the barrier function recovery of the skin wound, and the results demonstrated that hADSC-Exo increased the 
expression levels of some skin barrier proteins, like Filaggrin, Loricrin, and AQP3, suggested that hADSC-Exo is beneficial for promoting skin elasticity and barrier integrity. These findings provide strong in vitro research evidence that hADSC-Exo has a prospective clinical utility ability in wound healing and regeneration.

For local skin wounds, hADSC-Exo-sm showed markedly shorten healing time and enhanced re-epithelialization. However, the hADSC-Exo-sm alone is inadequate for the treatment of extensive burns and scalds. So, hADSC-Exosm combined therapy with the intravenous injection of hADSC-Exo and hADSC was necessary for clinical extensive burns and scalds. We administered hADSC-Exo to the skin wound of mice via intravenous injection, local smearing, and local injection. The data illustrated that the mice treated with exosomes healed faster than the control mice suggested that Hadsc-Exo enhance cutaneous wound healing. Of note, intravenous injection, and local smearing demonstrated superior wound healing ability relative to local injection, and we attributed this to the loss of exosomes during the local injection. Besides, when directly injecting exosomes into the wound, the wound can be inevitably disturbed, hence disturbing the wound healing process. After gaining entry into blood circulation, exosomes could be mobilized to the injured site and enhance wound healing. However, in addition to these, when intravenous injection of exosomes together with hADSC, a better efficacy was observed in wound healing, which may be attributed to the interaction between hADSC-Exo and hADSC. hADSC-Exo created the local microenvironment for hADSC living in vivo, and meanwhile, the living hADSC would release more exosomes to repair the wound.

\section{Conclusion}

In summary, hADSC can effectively promote skin wound healing while inhibiting scar formation at the wound. As a communication tool between cells, exosomes derived from hADSC can effectively replace hADSC as effective drugs for skin wound repair. Especially for extensive burns, trauma, or ulcers of various etiology, the combined treatment with the intravenous injection of hADSC-Exo and hADSC supplemented with the local application of smearing hADSC-Exo would be one of the best treatment options for clinical patients. Our study provides sufficient evidence and ideas for the clinical treatment of acute or chronic wounds.

\footnotetext{
Abbreviations

hADSC: Human adipose-derived mesenchymal stem cells; hADSCExo: Human adipose-derived mesenchymal stem cell-derived exosomes; PCNA: Proliferating cell nuclear antigen; VEGF: Vascular endothelial growth factor; TNF-alpha: Transforming growth factor alpha; TEM: Transmission electron microscopy; NTA: Nanosight tracking analysis; PBS: Phosphatebuffered saline; SEM: Scanning electron microscopy
}

\section{Supplementary Information}

The online version contains supplementary material available at https://doi. org/10.1186/s13287-021-02287-9.

Additional file 1: Supplementary material. The complete WB pictures of CD9 and CD63.

\section{Acknowledgements}

We thank the Base of Stem Cell Translational Medicine of Shanghai East Hospital for their assistance and guidance for hADSC culture.

\section{Authors' contributions}

Conceived and designed the experiments: $Y Z$ and BZ. Performed the experiments: YZ, BZ, XLZ, YJL, and STL. Analyzed the data: JC, YF, LL, NYZ, and PXL. Wrote the paper: YZ, JZ and JZ. The authors read and approved the final manuscript.

\section{Funding}

This work was supported by the National Natural Science Foundation of China (81771417, 31371379, and 31271120), the National Key Research and Development Program of China [2016YFA0700800], and the Peak Disciplines (Type IV) of Institutions of Higher Learning in Shanghai.

Availability of data and materials

Data and reagents will be provided upon availability and reasonable request.

\section{Declarations}

Ethics approval and consent to participate

All human samples and animal studies were approved by the Committee of Ethics on Experimentation of Tongji University. The experiments were conducted in accordance with the National Institutes of Health guidelines for the care and use of animals.

\section{Consent for publication}

Not applicable.

\section{Competing interests}

The authors declare that they have no competing interests.

\section{Author details}

${ }^{1}$ Research Center for Translational Medicine, Shanghai East Hospital, School of Medicine, Tongji University, Shanghai 200092, China. ${ }^{2}$ Key Laboratory of Spine and Spinal Cord Injury Repair and Regeneration of Ministry of Education, Stem Cell Translational Research Center of Tongji Hospital, School of Life Science and Technology, Tongji University, 389 Xincun Road, Shanghai 200065, China. 3Shanghai Institute of Stem Cell Research and Clinical Translation, Shanghai, China. ${ }^{4}$ Tongji Lifeng Institute of Regenerative Medicine, Tongji University, Shanghai, China.

Received: 25 September 2020 Accepted: 15 March 2021

Published online: 01 May 2021

\section{References}

1. Jeschke MG, Patsouris D, Stanojcic M, Abdullahi A, Rehou S, Pinto R, Chen $P$, Burnett M, Amini-Nik S. Pathophysiologic response to burns in the elderly. EBioMedicine. 2015;2(10):1536-48. https://doi.org/10.1016/j. ebiom.2015.07.040.

2. Han G, Ceilley R. Chronic wound healing: a review of current management and treatments. Adv Ther. 2017;34(3):599-610. https://doi.org/10.1007/s1232 5-017-0478-y.

3. Bielefeld KA, Amini-Nik S, Alman BA. Cutaneous wound healing: recruiting developmental pathways for regeneration. Cell Mol Life Sci. 2013;70(12): 2059-81. https://doi.org/10.1007/s00018-012-1152-9.

4. Mustoe TA, O'Shaughnessy K, Kloeters O. Chronic wound pathogenesis and current treatment strategies: a unifying hypothesis. Plast Reconstr Surg. 2006;117(SUPPLEMENT):35S-41S. https://doi.org/10.1097/01.prs.0000225431. 63010.1b.

5. Hu ZC, Chen D, Guo D, Liang YY, Zhang J, Zhu JY, et al. Randomized clinical trial of autologous skin cell suspension combined with skin 
grafting for chronic wounds. Br J Surg. 2015;102(2):e117-23. https://doi. org/10.1002/bjs.9688.

6. Wu Q, Ji FK, Wang JH, Nan H, Liu DL. Stromal cell-derived factor 1 promoted migration of adipose-derived stem cells to the wounded area in traumatic rats. Biochem Biophys Res Commun. 2015;467(1):140-5. https:// doi.org/10.1016/j.bbrc.2015.09.097.

7. Hocking AM, Gibran NS. Mesenchymal stem cells: paracrine signaling and differentiation during cutaneous wound repair. Exp Cell Res. 2010;316(14): 2213-9. https://doi.org/10.1016/j.yexcr.2010.05.009.

8. Kim WS, Park BS, Sung JH. The wound-healing and antioxidant effects of adipose-derived stem cells. Expert Opin Biol Ther. 2009;9(7):879-87. https:// doi.org/10.1517/14712590903039684.

9. Park BS, Jang KA, Sung JH, Park JS, Kwon YH, Kim KJ, et al. Adipose-derived stem cells and their secretory factors as a promising therapy for skin aging. Dermatol Surg. 2008:34:1323-6.

10. Otero-Vinas M, Falanga V. Mesenchymal stem cells in chronic wounds: the spectrum from basic to advanced therapy. Adv Wound Care (New Rochelle). 2016;5:149-63.

11. Goodarzi P, Alavi-Moghadam S, Sarvari M, Tayanloo BA, Falahzadeh K, Aghayan $\mathrm{H}$, et al. Adipose tissue-derived stromal cells for wound healing. Adv Exp Med Biol. 2018;1119:133-49. https://doi.org/10.1007/5584_2018_220.

12. Liang $X$, Ding $Y$, Zhang $Y$, Tse HF, Lian Q. Paracrine mechanisms of mesenchymal stem cell-based therapy: current status and perspectives. Cell Transplant. 2014;23(9):1045-59. https://doi.org/10.3727/096368913X667709.

13. Lu K, Li HY, Yang K, Wu JL, Cai XW, Zhou Y, Li CQ. Exosomes as potential alternatives to stem cell therapy for intervertebral disc degeneration: in-vitro study on exosomes in interaction of nucleus pulposus cells and bone marrow mesenchymal stem cells. Stem Cell Res Ther. 2017;8(1):108. https:// doi.org/10.1186/s13287-017-0563-9.

14. Colombo M, Raposo G, Thery C. Biogenesis, secretion, and intercellular interactions of exosomes and other extracellular vesicles. Annu Rev Cell Dev Biol. 2014;30(1):255-89. https://doi.org/10.1146/annurev-cellbio-101512-122326.

15. De Jong OG, Van Balkom BW, Schiffelers RM, Bouten CV, Verhaar MC. Extracellular vesicles: potential roles in regenerative medicine. Front Immunol. 2014;5:608.

16. Liu X, Wang S, Wu S, Hao Q, Li Y, Guo Z, Wang W. Exosomes secreted by adipose-derived mesenchymal stem cells regulate type I collagen metabolism in fibroblasts from women with stress urinary incontinence. Stem Cell Res Ther. 2018;9(1):159. https://doi.org/10.1186/s13287-018-0899-9.

17. Zhang $G$, Wang $Y$, Deng Z. Research progress of mechanisms of mesenchymal stem cells-derived exosomes in tissue repair. Zhongguo Xiu Fu Chong Jian Wai Ke Za Zhi. 2016;30:499-503.

18. Szatanek R, Baj-Krzyworzeka M, Zimoch J, Lekka M, Siedlar M, Baran J. The methods of choice for extracellular vesicles (EVs) characterization. Int J Mol Sci. 2017;18(6). https://doi.org/10.3390/ijms18061153.

19. Zhang W, Bai X, Zhao B, Li Y, Zhang Y, Li Z, Wang X, Luo L, Han F, Zhang J, Han S, Cai W, Su L, Tao K, Shi J, Hu D. Cell-free therapy based on adipose tissue stem cell-derived exosomes promotes wound healing via the PI3K Akt signaling pathway. Exp Cell Res. 2018;370(2):333-42. https://doi.org/10.1 016/j.yexcr.2018.06.035.

20. Chen $\mathrm{KH}$, Chen $\mathrm{CH}$, Wallace $\mathrm{CG}$, Yuen $\mathrm{CM}$, Kao GS, Chen YL, Shao PL, Chen YL, Chai HT, Lin KC, Liu CF, Chang HW, Lee MS, Yip HK. Intravenous administration of xenogenic adipose-derived mesenchymal stem cells (ADMSC) and ADMSCderived exosomes markedly reduced brain infarct volume and preserved neurological function in rat after acute ischemic stroke. Oncotarget. 2016;7(46): 74537-56. https://doi.org/10.18632/oncotarget.12902.

21. Shukla L, Yuan Y, Shayan R, Greening DW, Karnezis T. Fat therapeutics: the clinical capacity of adipose-derived stem cells and exosomes for human disease and tissue regeneration. Front Pharmacol. 2020;11:158. https://doi. org/10.3389/fphar.2020.00158.

22. Burger D, Vinas JL, Akbari S, Dehak H, Knoll W, Gutsol A, et al. Human endothelial colony-forming cells protect against acute kidney injury: role of exosomes. Am J Pathol. 2015;185(8):2309-23. https://doi.org/10.1016/.ajpath.2015.04.010.

23. Chang $\mathrm{CL}$, Chen HH, Chen KH, Chiang JY, Li YC, Lin HS, et al. Adiposederived mesenchymal stem cell-derived exosomes markedly protected the brain against sepsis syndrome induced injury in rat. Am J Transl Res. 2019; 11:3955-71.

24. Chang $\mathrm{CL}$, Sung $\mathrm{PH}$, Chen $\mathrm{KH}$, Shao PL, Yang $\mathrm{CC}$, Cheng $\mathrm{BC}$, et al. Adiposederived mesenchymal stem cell-derived exosomes alleviate overwhelming systemic inflammatory reaction and organ damage and improve outcome in rat sepsis syndrome. Am J Transl Res. 2018;10:1053-70.
25. Xu B, Chen SS, Liu MZ, Gan CX, Li JQ, Guo GH. Stem cell derived exosomesbased therapy for acute lung injury and acute respiratory distress syndrome: a novel therapeutic strategy. Life Sci. 2020;254:117766. https://doi.org/10.101 6/j.lfs.2020.117766.

26. Sun X, Jung JH, Arvola O, Santoso MR, Giffard RG, Yang PC, Stary CM. Stem cell-derived exosomes protect astrocyte cultures from in vitro ischemia and decrease injury as post-stroke intravenous therapy. Front Cell Neurosci. 2019;13:394. https://doi.org/10.3389/fncel.2019.00394.

27. Zhang J, Guan J, Niu X, Hu G, Guo S, Li Q, Xie Z, Zhang C, Wang Y. Exosomes released from human induced pluripotent stem cells-derived MSCs facilitate cutaneous wound healing by promoting collagen synthesis and angiogenesis. J Transl Med. 2015;13(1):49. https://doi.org/10.1186/s12 967-015-0417-0.

28. Wang T, Gu Q, Zhao J, Mei J, Shao M, Pan Y, Zhang J, Wu H, Zhang Z, Liu F. Calcium alginate enhances wound healing by up-regulating the ratio of collagen types I/III in diabetic rats. Int J Clin Exp Pathol. 2015;8(6):6636-45.

29. Volk SW, Wang Y, Mauldin EA, Liechty KW, Adams SL. Diminished type III collagen promotes myofibroblast differentiation and increases scar deposition in cutaneous wound healing. Cells Tissues Organs. 2011;194(1): 25-37. https://doi.org/10.1159/000322399.

30. Monavarian M, Kader S, Moeinzadeh S, Jabbari E. Regenerative scar-free skin wound healing. Tissue Eng Part B Rev. 2019;25(4):294-311. https://doi.org/1 0.1089/ten.teb.2018.0350.

31. Maharlooei MK, Bagheri M, Solhjou Z, Jahromi BM, Akrami M, Rohani L, Monabati A, Noorafshan A, Omrani GR. Adipose tissue derived mesenchymal stem cell (AD-MSC) promotes skin wound healing in diabetic rats. Diabetes Res Clin Pract. 2011;93(2):228-34. https://doi.org/10.1016/j.dia bres.2011.04.018.

32. Garcia-Olmo D, Herreros D, Pascual I, Pascual JA, Del-Valle E, Zorrilla J, et al. Expanded adipose-derived stem cells for the treatment of complex perianal fistula: a phase II clinical trial. Dis Colon Rectum. 2009;52(1):79-86. https:// doi.org/10.1007/DCR.0b013e3181973487.

33. Cho BS, Kim JO, Ha DH, Yi YW. Exosomes derived from human adipose tissue-derived mesenchymal stem cells alleviate atopic dermatitis. Stem Cell Res Ther. 2018;9(1):187. https://doi.org/10.1186/s13287-018-0939-5.

34. Wu CX, Liu ZF. Proteomic profiling of sweat exosome suggests its involvement in skin immunity. J Invest Dermatol. 2018;138(1):89-97. https:// doi.org/10.1016/j.jid.2017.05.040.

35. Kobayashi H, Ebisawa K, Kambe M, Kasai T, Suga H, Nakamura K, Narita Y, Ogata A, Kamei Y. Editors' choice effects of exosomes derived from the induced pluripotent stem cells on skin wound healing. Nagoya J Med Sci. 2018:80(2):141-53. https://doi.org/10.18999/nagjms.80.2.141.

36. Kim S, Lee SK, Kim H, Kim TM. Exosomes secreted from induced pluripotent stem cell-derived mesenchymal stem cells accelerate skin cell proliferation. Int J Mol Sci. 2018;19(10). https://doi.org/10.3390/ijms19103119.

37. Wang S, Hou Y, Li X, Song Z, Sun B, Li X, et al. Comparison of exosomes derived from induced pluripotent stem cells and mesenchymal stem cells as therapeutic nanoparticles for treatment of corneal epithelial defects. Aging (Albany NY). 2020;12:19546-62.

38. Cartwright P, McLean C, Sheppard A, Rivett D, Jones K, Dalton S. LIF/ STAT3 controls ES cell self-renewal and pluripotency by a Mycdependent mechanism. Development. 2005;132(5):885-96. https://doi. org/10.1242/dev.01670.

39. Xin Y, Wang YM, Zhang H, Li J, Wang W, Wei YJ, Hu SS. Aging adversely impacts biological properties of human bone marrow-derived mesenchymal stem cells: implications for tissue engineering heart valve construction. Artif Organs. 2010;34(3):215-22. https://doi.org/10.1111/j.152 5-1594.2009.00824.x.

40. Zaim M, Karaman S, Cetin G, Isik S. Donor age and long-term culture affect differentiation and proliferation of human bone marrow mesenchymal stem cells. Ann Hematol. 2012;91(8):1175-86. https://doi.org/10.1007/s00277012-1438-X.

41. Jeong JH. Adipose stem cells and skin repair. Curr Stem Cell Res Ther. 2010; 5(2):137-40. https://doi.org/10.2174/157488810791268690.

42. Gaur M, Dobke M, Lunyak W. Mesenchymal stem cells from adipose tissue in clinical applications for dermatological indications and skin aging. Int J Mol Sci. 2017;18(1). https://doi.org/10.3390/ijms18010208.

\section{Publisher's Note}

Springer Nature remains neutral with regard to jurisdictional claims in published maps and institutional affiliations. 